\title{
Comparison of Helicobacter Pylori Genotypes Obtained from the Oropharynx and Stomach of the Same Individuals - A Pilot Study
}

Lukeš P. ${ }^{1}$, Pavlík E. ${ }^{2}$, Potužníková B. ${ }^{2}$, Plzák J. ${ }^{1,3}$, Nártová E. ${ }^{1}$, Doseděl J. ${ }^{4}$, Katra R. ${ }^{5}$, Šterzl I. ${ }^{2}$, Betka J. ${ }^{1}$,Astl J. ${ }^{1}$

1Department of Otorhinolaryngology, Head and Neck Surgery, First Faculty of Medicine, Charles University in Prague and University Hospital Motol, Prague, Czech Republic;

Institute of Immunology and Microbiology, First Faculty of Medicine, Charles University in Prague and General University Hospital in Prague, Prague, Czech Republic;

${ }^{3}$ Institute of Anatomy, First Faculty of Medicine, Charles University in Prague, Prague, Czech Republic;

${ }^{4}$ Department of Gastroenterology, Hospital of Merciful Sisters of St. Charles Borromeo, Prague, Czech Republic;

${ }^{5}$ Department of Otorhinolaryngology, Head and Neck Surgery, Second Faculty of Medicine, Charles University in Prague and University Hospital Motol, Prague, Czech Republic

Received March 3, 2012; Accepted June 25, 2012.

Key words: Helicobacter pylori - Real-time PCR - Genotyping - Oropharynx Stomach - Comparison

Abstract: Helicobacter pylori has been recently detected in the oral cavity and oropharynx. However, the role it plays in oral and oropharyngeal pathogenesis remains unclear. The virulence of $H$. pylori strains can be distinguished according to the virulence factors genes carried. Our research has been focused on realtime PCR analysis of cagA and vacA genes of $H$. pylori strains in tonsils and tonsillar squamous cell cancer and their comparison with $H$. pylori strains obtained from

This study was supported by grant NT 11523 of the Internal Grant Agency of the Ministry of Health of the Czech Republic.

Mailing Address: Petr Lukeš, MD., PhD., Department of Otorhinolaryngology, Head and Neck Surgery, First Faculty of Medicine, Charles University in Prague and University Hospital Motol,V úvalu 84, 15006 Prague 5, Czech Republic; Phone: +420 224434 301; Fax: +420 224434 319; e-mail: petr.luk@gmail.com 
the gastric mucosa of the same patients. Urea breath test (UBT) test was used to detect a gastric $H$. pylori infection in 20 patients with previously proven $H$. pylori in the oropharynx. Genotyping of $\mathrm{H}$. pylori in gastric biopsies was performed in patients with positive gastric infection. Out of 20 patients positive for oropharyngeal $H$. pylori, 8 were positive for concurrent gastric $H$. pylori infection. In 6 of them gastric biopsies were obtained. Comparison of oropharyngeal and stomach $H$. pylori genotypes showed important differences. Four of 6 patients had different $H$. pylori strains in the oropharynx and stomach. The differences were found in cagA gene as well as in vacA gene. The finding of oral presence of $H$. pylori without concurrent stomach infection was confirmed using UBT. The results show that more than one $H$. pylori strain can be present in oropharynx and stomach in the same patient. The oropharyngeal infection seems to be independent to the gastric infection.

\section{Introduction}

Helicobacter pylori is a well known gastric pathogen (Peterson, 1991; Israel and Peek, 2001). H. pylori is a declared type I carcinogen by IARC (1994). Since it was detected in the oral cavity (Nguyen et al., 1993; Riggio and Lennon, 1999; Karczewska et al., 2002) and oropharynx (Bulut et al., 2006; Pavlik et al., 2007; Abdel-Monem et al., 2011) a hypothesis about its possible influence in oropharyngeal pathogenesis is being considered (Rubin et al. 2003; Lukes et al., 2008). H. pylori is spread from person to person by oral-oral or faecal-oral route (Brown, 2000). Assuming the oral cavity and oropharynx as a gateway of infection, we can expect that in the oral cavity and oropharynx of the same individual we should find $H$. pylori strains of the same genotype. The virulence of $H$. pylori strains differs according to the production of several virulence factors. The most important virulence factors, which were described as associated with gastric pathogenesis, are CagA (cytotoxic associated gene A) and VacA (vacuolizating cytotoxin A). Genome sequence analysis of $H$. pylori DNA led to the identification of genes encoding these virulence factors. The immunodominant CagA protein is encoded by the group of genes called the cagA-PAI (pathogenicity island), which is present in 60-70\% of strains (Mobley, 1996). H. pylori cagA+ strains are associated with a significantly increased risk for severe gastritis, atrophic gastritis, peptic ulcer disease and distal gastric cancer compared with cagA- strains (Portal-Celhay and Perez-Perez, 2006). All $H$. pylori strains carry the vacA gene. Significant polymorphism of this gene exists. VacA alleles possess one of two types of signal region, s1 or s2, and one of two mid-regions, $\mathrm{m} 1$ or $\mathrm{m} 2$, occurring in all possible combinations. $H$. pylori strains with different forms of vacA have different pathogenetic potential (Portal-Celhay and Perez-Perez, 2006). The question whether the finding of oropharyngeal $H$. pylori indicates permanent colonization or it is only a sign of contamination of this area by gastric juices still remains unclear. 
The presence of different genotypes of $H$. pylori in the oral cavity and stomach is now being considered (Wang et al., 2002; Loster et al., 2006). The comparison of oropharyngeal and stomach genotypes has not previously been studied. The aim of this study was to find out whether one person could host different $H$. pylori strains in different locations. The other aim was to determine whether the oropharyngeal presence could be caused by contamination by gastric contents or it is a permanent colonization which could be independent from gastric infection. Previous research in Department of Otorhinolaryngology, Head and Neck Surgery, First Faculty of Medicine, Charles University in Prague, has been focused on real-time PCR analyzing cagA and vacA genotypes of $H$. pylori strains in tonsils and tonsillar squamous cell cancer (Lukes, 2010). In 6 cases we have compared the DNA sequences of $H$. pylori strains obtained from oropharyngeal area with the strains obtained from gastric mucosa of the same patients.

\section{Material and Methods}

Collection of clinical specimens

The study was approved by the appropriate ethics committee, and informed written consent was obtained from every participant.

Twenty patients with previously proven $H$. pylori in the oropharynx were selected for routine urea breath testing (UBT) for the presence of $H$. pylori in the stomach. The patients were part of a study on the detection of oropharyngeal $H$. pylori (Lukes, 2010). They underwent surgery and subsequently the tissue samples were analysed for the presence of $H$. pylori. Indications for surgery were tonsillar spinocellular cancer (SCC) (9 patients), obstructive sleep apnea syndrome (OSAS) (5 patients) and chronic tonsillitis (6 patients).

The tonsillar specimens were collected using sterile instruments at the beginning of surgery, immediately after insertion of endotracheal tube, prior to application of local anaesthetics or disinfection substances into the oral cavity. Biopsies were immersed into Microtest ${ }^{R}$ M4RT transport media (Remel Inc., USA) and transported to the laboratory for real-time PCR analysis. After positive results for $H$. pylori presence in tonsillar tissue, the presence of gastric infection was confirmed using UBT.

Eight patients were found positive for $H$. pylori by UBT. Six of them ( 3 with cancer, 2 with OSAS and 1 with tonsillitis) gave their consent for gastroscopy (remnant two patients declined any further examination). The gastroscopy was performed subsequently at the Department of Gastroenterology, Hospital of Merciful Sisters of St. Charles Borromeo, Prague. Gastric mucosa samples were taken from the antrum and body of the stomach and immersed in Microtest ${ }^{R}$ M4RT transport media for real-time PCR analysis. The genotyping of gastric strains was performed. The gastric genotypes were compared to oropharyngeal genotypes in the same individuals. 
Preparation of genomic DNA

$H$. pylori DNA isolation was performed on MagNA Pure Compact System

(Tegimenta AG, Rotkreuz, Switzerland) using Protocol "Total_NA 400_100” and MagNA Pure Compact Nucleic Acid Isolation Kit I (Roche Diagnostics, Basel,

Switzerland) with pre-treatment in MagNA Pure Bacteria Lysis Buffer (Roche Diagnostics, Basel, Switzerland). Isolated nucleic acid specimens were stored in $-80{ }^{\circ} \mathrm{C}$ until genotyping assays were performed.

\section{$P C R$ amplification and genotyping}

For genotyping, 3 real-time PCR TaqMan assays had been developed in cooperation with TIB-Molbiol Berlin, Germany; one for the cagA gene, the second for the vacA gene middle region and the last one for the vacA gene signal region. Primers used for PCR detection of $H$. pylori were: cagA $F$ (sense), cagA R (antisense), HPMGF+ (sense), HPMGR- (antisense), VA1F (sense) and VA1R- (antisense) according to Atherton et al. (1995) and van Doorn et al. (1998, 1999) (Table 1).

TaqMan hybridisation probes were developed for cagA, vacA $m 1$ and vacA $m 2$ specific sequence detection (Table 2). For the cagA assay FAM-BBQ labelled probe was used (detection $530 \mathrm{~nm}$ ), for vacA middle region assay FAM-BBQ labelling was used for M1 TM-probe (530 nm) and HEX-BBQ labelling for M2 TM-probe $(560 \mathrm{~nm})$.

$\mathrm{TaqMan}^{\mathrm{R}}$ real-time PCR assays were run on a LightCycler ${ }^{R}$ instrument, version 2.0 (six channel detection: 530, 570, 610, 640, 670 and 705 nm). Commercial LightCyclerR TaqMan R Master (Roche Applied Science, Basel, Switzerland) was used $-15 \mu$ of MasterMix including primers and probes and $5 \mu$ l of sample DNA isolate per $20 \mu$ l capillary.

For real-time PCR for vacA gene signal region hybridisation probes S1a LC (LC610), S1b LC (LC640) and S2 LC (LC705) were used together with commercial LightCycler ${ }^{R}$ FastStart DNA Master PLUS HybProbe (Roche Diagnostics, Basel, Switzerland) $-15 \mu \mathrm{l}$ of MasterMix including primers and probes and $5 \mu \mathrm{l}$ of sample DNA isolate per $20 \mu$ l capillary.

The real-time PCR assay program consisted of 45 cycles with an initial denaturation step at $95^{\circ} \mathrm{C}$ for 10 minutes in first cycle and than for $10 \mathrm{~s}$ in remnant cycles. Annealing temperatures were selected 58 and $68{ }^{\circ} \mathrm{C}$ for 10,15 or $20 \mathrm{~s}$ according to used probes. An extension step at $72{ }^{\circ} \mathrm{C}$ for $45 \mathrm{~s}$ was followed by real-time detection at proper wavelength according to used probe.

\section{Results}

Only 8 of 20 patients with proven oropharyngeal presence of $H$. pylori showed concurrent presence of gastric infection by UBT.

In gastric biopsies the presence of $H$. pylori was proven by real-time PCR in all 6 patients who underwent endoscopy. Endoscopic examination showed mucosal changes typical for gastric inflammation in four patients, the inflammation was 
clinically asymptomatic. None of the patients admitted any gastroduodenal complaints.

Comparison of gastric and oropharyngeal genotypes showed important differences. Results are shown in Table 3. Three patients (nos. 1, 2 and 5) had the cagA positive $H$. pylori strain in the stomach but negative in the oropharynx. Two of them also showed differences in the vacA gene $S$ region (nos. 1 and 5). Patient no. 6 showed a difference in S region alone. In two patients (nos. 3 and 4) the $H$. pylori strains from the oropharynx and stomach were identical.

Table 1 - PCR primers for amplification of cagA and vacA sequences

\begin{tabular}{|c|c|c|c|}
\hline Gene & Primer & $\begin{array}{c}\text { No. of } \\
\text { nucleotides }\end{array}$ & Sequence \\
\hline \multirow[t]{2}{*}{ cagA } & cagA F & 24 & 5'-TTG ACC AAC AAC CAC AAA CCG AAG-3' \\
\hline & cagA R & 22 & 5'-CTT CCC TTA ATT GCG AGA TTC C-3' \\
\hline \multirow[t]{2}{*}{ vacAs } & VAIF & 21 & 5'-ATG GAA ATA CAA CAA ACA CAC-3' \\
\hline & VAIR & 19 & 5'-CTG CTT GAA TGC GCC AAA C-3' \\
\hline \multirow[t]{2}{*}{$\operatorname{vac} A \mathrm{~m}$} & HPMGF & 21 & 5'-CAG AGC CAC TTT CAA TAA CGA-3' \\
\hline & HPMGR & 21 & 5'-CGT CCA AAT AAT TCC AAG GG-3' \\
\hline
\end{tabular}

Table 2 - PCR probes for detection of cagA and vacA sequences

\begin{tabular}{ccccc}
\hline Gene & Type & $\begin{array}{c}\text { No. of } \\
\text { nucleotides }\end{array}$ & \multicolumn{1}{c}{ Sequence } & $\begin{array}{c}\text { Detection } \\
(\mathrm{nm})\end{array}$ \\
\hline cagA & cag_TM & 28 & $\begin{array}{l}\text { 6FAM-ATA ACG CTG TCG CTT CAT ACG } \\
\text { ATC CTG A-BBQ }\end{array}$ & 530 \\
\hline vacA s & SIa_LC & 21 & $\begin{array}{l}\text { LC Red6I0-GCR TTR GTC AGC ATC ACA } \\
\text { CCG-PH }\end{array}$ & 610 \\
& SIb_LC & 21 & $\begin{array}{l}\text { LC Red640-GCG TTG ATT AGY KCC ATA } \\
\text { CCG-PH }\end{array}$ & 640 \\
& S2_LC & 21 & $\begin{array}{l}\text { LC Red705-GCT AAY ACG CCA AAY GAT } \\
\text { CCC-PH }\end{array}$ & 705 \\
\hline vacA m & MI_TM & 30 & $\begin{array}{l}\text { 6FAM-ACC ACC ATT ACC CGT ATC AAT } \\
\text { ACCTTT AAA-BBQ }\end{array}$ & 530 \\
\cline { 2 - 5 } & M2_TM & 26 & $\begin{array}{l}\text { HEX-CTA GTG TTT AGC CCG TTA TCG } \\
\text { CTCTT-BBQ }\end{array}$ & 560 \\
\hline
\end{tabular}


Table 3 - Results of Helicobacter pylori investigation

\begin{tabular}{ccccc}
\hline No. & Diagnosis & Oropharyngeal genotype & Gastric genotype & Gastritis \\
\hline 1. & tonsillar cancer & cagA-S1aM1 & cagA+S1a/bM1 & - \\
2. & tonsillar cancer & cagA-S1bM2 & cagA+S1bM2 & - \\
3. & tonsillar cancer & cagA+S1bM2 & cagA+S1bM2 & + \\
4. OSAS & cagA-S1aM2 & cagA-S1aM2 & + \\
5. OSAS & cagA-S1aM2 & cagA+S1a/bM2 & + \\
6. & tonsillitis & cagA+S1bM1 & cagA+S1aM1 & + \\
\hline
\end{tabular}

OSAS - obstructive sleep apnea syndrome

\section{Discussion}

The results of the present study showed that oropharyngeal lymphatic tissue can harbour $\mathrm{H}$. pylori strains independently to the stomach infection. Real-time PCR genotyping of oropharyngeal and gastric strains in patients with present infection in both areas showed that different $H$. pylori strains can be found in oropharynx and stomach of the same individual. Four of 6 investigated patients had different $H$. pylori strains in oropharynx and stomach. The differences were found in cagA gene as well as in vacA gene.

These results are in concordance with the first study that compared cytotoxin genotypes of $\mathrm{H}$. pylori between the stomach and oral cavity published by Wang et al. (2002). Authors used saliva for genotyping of oral $H$. pylori. They showed that more than one $H$. pylori strain may exist in the saliva and the stomach of the same individual.According to our knowledge, our study is the first to compare H. pylori genotypes between the stomach and tonsillar tissue biopsies. In this study the patients were selected according to the oropharyngeal $H$. pylori infection. None of them admitted any gastrointestinal complaints. Gastroscopy showed the presence of gastritis in the majority of patients although all were clinically asymptomatic. This differs from previous studies, which were primarily focused on patients with gastric disease. Only a few other studies focused on comparison of $H$. pylori strains in the oral cavity and stomach. Shames et al. (1989), Khandaker et al. (1993), Zhang and Lu (1997) and Hu et al. (2002) showed evidence, that both sites are infected with the same $H$. pylori strain. These authors used a DNA fingerprinting method, PCR adopted single strand conformation polymorphism (SSCP) or restriction endonuclease analysis respectively. None of these studies compared the different gene sequences of VacA gene. The variance of our results could be explained by different diagnostic methods used. Nevertheless real-time PCR genotyping is considered to be highly specific with high degree of reliability (Burgers et al., 2008).

Another study that used real-time PCR to detect $H$. pylori in the oral cavity (Martinez-Gomis et al., 2006) did not find any individual positive for oral $H$. pylori. 
The authors did not mention in their paper the use of any transport medium for preserving $H$. pylori DNA, which seems to be very important for successful PCR detection (Pavlik et al., 2007).

The presence of oropharyngeal $H$. pylori without concurrent stomach infection was confirmed using UBT and is in accordance with previously published data by Burgers et al. (2008), who showed that $H$. pylori can be present in the oral cavity independently of stomach colonization. UBT has proved to be one of the most accurate methods for assessing gastric $H$. pylori status (Gisbert and Pajares, 2004). Loster et al. (2006) used a modified UBT for detecting oral $H$. pylori. The solution containing of ${ }^{13} \mathrm{C}$-urea was kept in the oral cavity for 5 min to detect oral urease producing bacterium. When using a standard UBT procedure, the solution of ${ }^{13} \mathrm{C}$-urea is swallowed immediately, so the time of contact with oropharyngeal mucosa is too short detect oropharyngeal $H$. pylori.

The finding of presence of $H$. pylori in the oropharyngeal tissue independently from the gastric infection confirms that $H$. pylori can permanently colonize oropharyngeal tissue and the oropharyngeal presence is not caused only by contamination by gastric contents. This is further confirmed by the finding of different strains in the stomach and oropharynx in the same individuals.

On the other hand the results re-open the question of $H$. pylori transmission. When we consider the oral cavity and oropharynx as a gateway for infection of stomach, we would expect to find identical strains in both areas. This inconsistency must be resolved by further analysis.

Although $H$. pylori is proved to be highly pathogenic bacterium with carcinogenic potential, the data about oropharyngeal pathogenesis are insufficient. Culture of $H$. pylori from oral and oropharyngeal samples is extremely difficult (reviewed by Lukes et al., 2008). PCR techniques are not able to solve the question of viability of bacterial cells. The lower number of cagA positive oropharyngeal strains compared to gastric strains could shed some light on the question of oropharyngeal pathogenesis, nevertheless the number of investigated patients is very small. Further improvement of investigation methods such as culture is needed for obtaining more information about possible involvement of $H$. pylori in oropharyngeal diseases.

\section{References}

Abdel-Monem, M. H., Magdy, E. A., Nour, Y.A., Harfoush, R. A., Ibreak, A. (2011) Detection of Helicobacter pylori in adenotonsillar tissue of children with chronic adenotonsillitis using rapid urease test, PCR and blood serology: a prospective study. Int. J. Pediatr. Otorhinolaryngol. 75, 568-572.

Atherton, J. C., Cao, P., Peek, R. M. Jr., Tummuru, M. K., Blaser, M. J., Cover, T. L. (1995) Mosaicism in vacuolating cytotoxin alleles of Helicobacter pylori. Association of specific vacA types with cytotoxin production and peptic ulceration. J. Biol. Chem. 270, 17771-17777.

Brown, L. M. (2000) Helicobacter pylori: epidemiology and routes of transmission. Epidemiol. Rev. 22, $283-297$.

Bulut, Y., Agacayak, A., Karlidag, T., Toraman, Z. A., Yilmaz, M. (2006) Association of cagA+ Helicobacter pylori with adenotonsillar hypertrophy. Tohoku J. Exp. Med. 209, 229-233. 
Burgers, R., Schneider-Brachert, W., Reischl, U., Behr, A., Hiller, K. A., Lehn, N., Schmalz, G., Ruhl, S. (2008) Helicobacter pylori in human oral cavity and stomach. Eur.J. Oral Sci. 116, 297-304.

Gisbert, J. P., Pajares, J. M. (2004) Review article: C-urea breath test in the diagnosis of Helicobacter pylori infection - a critical review. Aliment. Pharmacol.Ther. 20, 1001-1017.

Hu,W., Cao, C., Meng, H., Zhang, J., Ma, D., Zhang, L. (2002) Detection and analysis of Helicobacter pylori in oral cavity and stomach from chronic gastritis patients. Zhonghua Yi Xue Za Zhi 82, 1037-1041. (in Chinese)

IARC (1994) Schistosomes, liver flukes and Helicobacter pylori. IARC Working Group on the Evaluation of Carcinogenic Risks to Humans. Lyon, 7-14 June 1994. IARC Monogr. Eval. Carcinog. Risks Hum. 61, 1-241.

Israel, D. A., Peek, R. M. (2001) Pathogenesis of Helicobacter pylori-induced gastric inflammation. Aliment. Pharmacol.Ther. 15, 1271-1290.

Karczewska, E., Konturek, J. E., Konturek, P. C., Czesnikiewicz, M., Sito, E., Bielanski,W., Kwiecien, N., Obtulowicz,W., Ziemniak, W., Majka, J., Hahn, E. G., Konturek, S. J. (2002) Oral cavity as a potential source of gastric reinfection by Helicobacter pylori. Dig. Dis. Sci. 47, 978-986.

Khandaker, K., Palmer, K. R., Eastwood, M. A., Scott, A. C., Desai, M., Owen, R. J. (1993) DNA fingerprints of Helicobacter pylori from mouth and antrum of patients with chronic ulcer dyspepsia. Lancet 342, 751.

Loster, B.W., Majewski, S.W., Czesnikiewicz-Guzik, M., Bielanski, W., Pierzchalski, P., Konturek, S. J. (2006) The relationship between the presence of Helicobacter pylori in the oral cavity and gastric in the stomach. J. Physiol. Pharmacol. 57, 91-100 (Suppl. 3).

Lukes, P. (2010) Expression of NO synthases, TGF-beta 1 and EGF in patients with documented oropharyngeal Helicobacter pylori infection and its role in the pathology of the oropharynx. Dissertation, Charles University in Prague.

Lukes, P., Astl, J., Pavlik, E., Potuznikova, B., Sterzl, I., Betka, J. (2008) Helicobacter pylori in tonsillar and adenoid tissue and its possible role in oropharyngeal carcinogenesis. Folia Biol. (Praha) 54, 33-39.

Martinez-Gomis, J., Diouf, A., Lakhssassi, N., Sixou, M. (2006) Absence of Helicobacter pylori in the oral cavity of 10 non-dyspeptic subjects demonstrated by real-time polymerase chain reaction. Oral. Microbiol. Immunol. 21, 407-410.

Mobley, H. L. (1996) Defining Helicobacter pylori as a pathogen: strain heterogeneity and virulence. Am. J. Med. 100, 2S-9S, discussion 9S-11S.

Nguyen, A. M., Engstrand, L., Genta, R. M., Graham, D.Y., el-Zaatari, F.A. (1993) Detection of Helicobacter pylori in dental plaque by reverse transcription-polymerase chain reaction. J. Clin. Microbiol. 31, 783-787.

Pavlik, E., Lukes, P., Potuznikova, B., Astl, J., Hrda, P., Soucek, A., Matucha, P., Dosedel, J., Sterzl, I. (2007) Helicobacter pylori isolated from patients with tonsillar cancer or tonsillitis chronica could be of different genotype compared to isolates from gastrointestinal tract. Folia Microbiol. (Praha) 52, 91-94.

Peterson,W. L. (1991) Helicobacter pylori and peptic ulcer disease. N. Engl. J. Med. 324, 1043-1048.

Portal-Celhay, C., Perez-Perez, G. I. (2006) Immune responses to Helicobacter pylori colonization: mechanisms and clinical outcomes. Clin. Sci. (Lond.) 110, 305-314.

Riggio, M. P., Lennon, A. (1999) Identification by PCR of Helicobacter pylori in subgingival plaque of adult periodontitis patients. J. Med. Microbiol. 48, 317-322.

Rubin, J. S., Benjamin, E., Prior, A., Lavy, J. (2003) The prevalence of Helicobacter pylori infection in malignant and premalignant conditions of the head and neck. J. Laryngol. Otol. 117, 118-121.

Shames, B., Krajden, S., Fuksa, M., Babida, C., Penner, J. L. (1989) Evidence for the occurrence of the same strain of Campylobacter pylori in the stomach and dental plaque. J. Clin. Microbiol. 27, 2849-2850.

van Doorn, L. J., Figueiredo, C., Rossau, R., Jannes, G., van Asbroek, M., Sousa, J. C., Carneiro, F., Quint, W. G. (1998) Typing of Helicobacter pylori vacA gene and detection of cagA gene by PCR and reverse hybridization. J. Clin. Microbiol. 36, 1271-1276.

Lukeš P. et al. 
van Doorn, L. J., Figueiredo, C., Megraud, F., Pena, S., Midolo, P., Queiroz, D. M., Carneiro, F., Vanderborght, B., Pegado, M. D., Sanna, R., De Boer, W., Schneeberger, P. M., Correa, P., Ng, E. K., Atherton, J., Blaser, M. J., Quint,W. G. (1999) Geographic distribution of vacA allelic types of Helicobacter pylori. Gastroenterology 116, 823-830.

Wang, J., Chi, D. S., Laffan, J. J., Li, C., Ferguson, D. A. Jr., Litchfield, P., Thomas, E. (2002) Comparison of cytotoxin genotypes of Helicobacter pylori in stomach and saliva. Dig. Dis. Sci. 47, 1850-1856.

Zhang, Y., Lu, X. (1997) Detection and differentiation of Helicobacter pylori from gastric biopsy and saliva by PCR-SSCP. Zhonghua Nei Ke Za Zhi 36, 446-449. (in Chinese) 